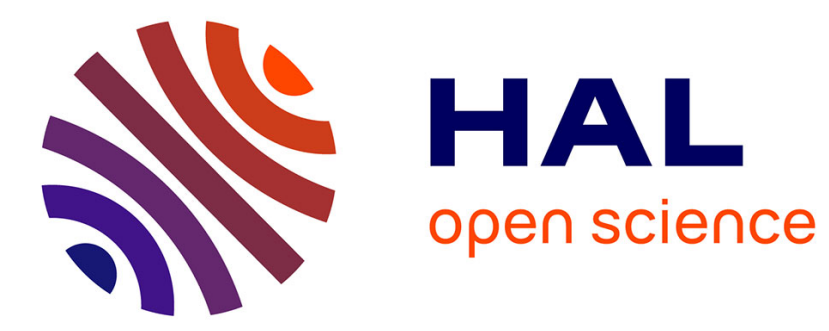

\title{
La pathologie du langage entre les lettres et la médecine (1880-1900)
}

Gabriel Bergounioux

\section{To cite this version:}

Gabriel Bergounioux. La pathologie du langage entre les lettres et la médecine (1880-1900). Communications, 1992, 54 (1), pp.229 - 239. 10.3406/comm.1992.1824 . halshs-01842485

\section{HAL Id: halshs-01842485 \\ https://shs.hal.science/halshs-01842485}

Submitted on 18 Jul 2018

HAL is a multi-disciplinary open access archive for the deposit and dissemination of scientific research documents, whether they are published or not. The documents may come from teaching and research institutions in France or abroad, or from public or private research centers.
L'archive ouverte pluridisciplinaire HAL, est destinée au dépôt et à la diffusion de documents scientifiques de niveau recherche, publiés ou non, émanant des établissements d'enseignement et de recherche français ou étrangers, des laboratoires publics ou privés. 

1900)

Gabriel Bergounioux

Citer ce document / Cite this document :

Bergounioux Gabriel. La pathologie du langage entre les lettres et la médecine (1880-1900). In: Communications, 54, 1992. Les débuts des sciences de l'homme. pp. 229-239;

doi : https://doi.org/10.3406/comm.1992.1824

https://www.persee.fr/doc/comm_0588-8018_1992_num_54_1_1824

Fichier pdf généré le 10/05/2018 


\section{La pathologie du langage entre les lettres et la médecine (1880-1900)}

L'institutionnalisation des sciences humaines au $\mathrm{XIX}^{\mathrm{e}}$ siècle ne laisse jamais mieux apparaître ses exigences et ses modalités que livrée aux luttes de concurrence scientifique, lorsque deux champs de production savants, impliquant des histoires, des sollicitations et des agents différents, s'affrontent dans la revendication d'un domaine, lui-même partagé entre les profits spécifiques de l'autonomie et la légitimité issue de la perpétuation de méthodes, d'un fonctionnement, voire d'un apparat (c'est-à-dire, en dernière instance, de rapports sociaux de production scientifique) antérieurs.

Ainsi la pathologie du langage emportait-elle, dès lors que son inclusion dans le champ scientifique se supportait, médicalement, de la transformation des interrogations sur le statut de la maladie mentale, une part conflictuelle avec le linguiste et, plus encore, le "psychologue ». Celui-ci ne peut rester indifférent, dans sa prétention nouvelle ', à un débat qui se noue dans les années 1880: à qui revient le préciput de la théorie du langage, du médecin aliéniste, du philosophe, du linguiste ou du psychologue?

Après un rapide récapitulatif concernant la question de l'aphasie dans les années 1860 et suivantes ${ }^{2}$, nous entendons retracer dans cet article le débat qui, opposant Victor Egger et Jean-Martin Charcot, devait donner à la Faculté de médecine l'autorité sur cette question sans que ni le linguiste ni le psychologue, dont les propositions ne manquaient pas d'incidence, voire de pertinence, ne puissent s'y imposer.

\section{Broca et la pathologie du langage.}

C'est peut-être à Broca que nous sommes redevables d'avoir, pour la première fois, une approche unifiée qui, pour un temps, réconcilia 
deux approches antagonistes. Face à l'hôpital où le clinicien pointait de son scalpel le cerveau détruit, le philosophe, souvent solitaire, suivant la longue tradition française de l'introspection (de Montaigne à Amiel, pour l'époque), prétendait interroger le fonctionnement de la pensée à l'œuvre et dans ses œuvres. Quel que soit le nom des théories (sensualisme, vitalisme, éclectisme...), toutes les configurations du travail intellectuel trouvaient à se disperser entre la pratique médicale et le travail réflexif du "penseur", cultivant la méditation et l'exactitude du bien-dire, et il y eut (rarement) des médecins spiritualistes comme il y avait (fort peu) des philosophes matérialistes ${ }^{3}$.

Avec Broca, l'aphasie - qu'il appelle " aphémie » - reçoit une assignation nosographique conforme au dogme «paralléliste» de la médecine française : qu'à toute affection (ou opération) mentale corresponde une affection (ou opération) physique. Élargissant les travaux de Bouillaud, Cruveilhier et Gratiolet, Broca s'attache à la question du désordre linguistique qu'il approprie définitivement, en 1863, à la clinique par l'autopsie et la localisation ${ }^{4}$. Significativement, l'annonce n'en est pas faite dans un amphithéâtre de Bicêtre mais, plus solennellement, devant les membres de la "Société d'anthropologie». Fort d'une dizaine de dissections, Broca lance la théorie du pied de la troisième circonvolution frontale gauche comme zone du langage articulé ${ }^{5}$. En dépit de contre-exemples et de discussions - notamment de la part de Charcot -, la définition de l'aphasie est figée à partir de 1865 : une maladie provoquée par une altération du cerveau, dont le symptôme est la désorganisation de la parole sans que les facultés mentales soient atteintes. La reformulation médicale se poursuivra à l'étranger, particulièrement en Allemagne ${ }^{6}$, cependant qu'en France Taine, en popularisant la doctrine associationniste, opère un partage entre l'œuvre médicale, dont il reconnaît la nécessité préalable, et le domaine du philosophe qui se réserve la théorie déductive et, au-delà, la doctrine morale et la typologie psychologique - une position qui sera celle de Ribot jusqu'en 1880. Les premiers essais de sémantique (ceux de Bréal dès 1868, par exemple ${ }^{7}$, n'aboutissent pas à des formulations de portée générale qui remettraient en cause cette hypothèse.

Pour qui observe le champ des sciences sociales en France au $\mathrm{XIX}^{\mathrm{e}}$ siècle, il semble que se dessinent deux crises entre 1860 et 1900 : de 1860 à 1880, une crise de constitution, provoquée par la linguistique, l'histoire et l'anthropologie (c'est le travail de Broca et de son école), bouleversant les équilibres acquis par les facultés de lettres autour d'un enseignement routinisé des humanités; de 1880 à 1900, une crise de redistribution provoquée par l'apparition de disciplines 
nouvelles à l'intérieur du champ universitaire : géographie, sociologie, pédagogie, psychologie... corrélativement à une redéfinition progressive des conceptions sociale et nationale ${ }^{8}$. A cette phase critique appartiendraient les travaux d'Egger et de Charcot. Cette recomposition du marché universitaire impliquait la reconduction de divisions acquises, comme en témoignerait la fondation de la psychologie, divisée dès l'origine entre Ribot et Egger (parmi d'autres).

\section{Egger et la parole intérieure.}

Victor Egger représente le type même de l'héritier au sens de Bourdieu ${ }^{9}$ : fils d'Emile Egger, l'un des maîtres de la philologie française entre 1840 et 1870 , il choisit la discipline la plus prestigieuse (la philosophie), à l'ENS comme à l'agrégation, occupant divers postes en lycée avant de recevoir, en 1882, la chaire de philosophie à l'université de Nancy.

Face au reflux des grandes synthèses philosophiques, mieux informé sur les travaux allemands et les disciplines d'avenir qui se constituent, Victor Egger s'intéresse immédiatement à la psychologie, pour laquelle il propose une approche contraire aux doctrines expérimentales de Ribot et au physiologisme sommaire de Luys ${ }^{10}$, élaborant une doctrine originale qui écarte le modèle du laboratoire et de la clinique en référence à la tradition des humanités et à l'introspection philosophique et autobiographique.

En 1881, Victor Egger soutient une thèse remarquée : La Parole intérieure. Remontant à des textes de l'Égypte pharaonique et suivant l'histoire de la pensée occidentale à travers la Grèce classique et Rome, Egger s'inscrit dans la tradition de la philosophie spiritualiste française, ne se reconnaissant de dette qu'à l'égard de De Bonald, Maine de Biran et Condillac, réfutant les doctrines de ce qu'il appelle l'«école du muscle» (Bain en Angleterre et Taine en France). Devancé par Ribot dans la création de la psychologie ${ }^{11}$, Egger se sépare de son devancier en prônant, face à une psychologie expérimentale, matérialiste, objectiviste, moderne (le reproche de "germanique »n'est pas loin), une autre psychologie, introspective et spiritualiste (Egger parle plus facilement de l'« âme»que de l'«esprit »), fidèle à une tradition "classique », nationale.

Institutionnellement aussi, Egger se sépare résolument de la médecine, dont il réduit la portée à la pathologie et à l'autopsie, tranchant sur la démarche de Ribot - auditeur assidu de Claude Bernard, Charcot, Destre, et qui fréquenta le laboratoire du docteur Robin. Par la 


\section{Gabriel Bergounioux}

filiation qu'il revendique, il requiert sa place, auprès de la philosophie, dans le prytanée des lettres, à l'Université d'abord, au Collège de France un jour ${ }^{12}$. La rivalité est d'autant plus tranchée que l'introspection est l'objet d'un refus catégorique et principiel de Ribot, plus nettement même dans l'introduction de sa Psychologie allemande contemporaine (1879) que dans sa Psychologie anglaise contemporaine. Est-ce le hasard des nominations qui livra à Egger la chaire de Nancy (la rivale de l'université allemande de Strasbourg), si importante du fait que Bernheim, un des maîtres français de Freud, y créait une école dont les prétentions en psychologie se fondaient sur un recours systématique à l'expérimentation hypnotique et à l'observation clinique? Quoi qu'il en soit, la thèse d'Egger rencontra un certain écho érudit que reflètent assez mal les comptes rendus qui en parurent ici ou là, proches souvent du contresens.

\section{Charcot et l'aphasie.}

Dès 1893, Paul Ajam ${ }^{13}$ a dû interroger Paul Blocq, le chef des travaux anatomo-pathologiques à la Salpêtrière, pour apprendre que Charcot avait traité la question de l'aphasie à partir du deuxième trimestre de l'année 1883. En effet, les Leçons sur les maladies du système nerveux, professées cette année-là et éditées par Charles Féré, s'interrompent brusquement à la neuvième : "Exemple d'une affection spinale. »

La suite ne sera jamais publiée en France; il n'en existe qu'une version italienne : Differenti forme d'Afasia, éditée par G. Rummo en 1884, et Lezione cliniche : malattie del sistema nervoso, publié par D. Miliotti en 1885. Ces éditions elles-mêmes sont lacunaires, sommaires (on y trouve quelques erreurs grossières) et, somme toute, difficilement accessibles à un lecteur français : pourtant, en occultant non l'enchaînement de ses arguments, mais leur exposition, Charcot assurera le triomphe de son enseignement, qui, pendant un quart de siècle, sera la vérité clinique.

Dans ses leçons, Charcot ne cesse de répéter qu'il s'agit d'un travail inachevé, multipliant les réticences devant ses propres résultats, laissant en suspens :

- le statut de la pensée par rapport au langage et à la mémoire ;

- le degré d'homologie des procédures non linguistiques (mimique, musique, calcul) avec le langage ;

- le nombre de centres sensitifs et moteurs à prendre en considération (oscillant entre deux et cinq à partir du moment où il intègre un «centre d'idéation », c'est-à-dire de synthèse) ${ }^{14}$; 
- le nombre de «types » à définir en fonction de leur dominance sensori-motrice (type visuel, type auditif ${ }^{15} \ldots$...

Quant aux observations, après avoir récusé Kussmaul qui n'en présenterait que d'empruntées, il en analyse soixante-quinze dont huit, au plus, sont originales.

Pour que les thèses de Charcot s'imposent, il n'était pas inutile que soit oubliée la fragilité des prémisses et qu'une vulgarisation procédant de l'évidence, celle de la compétence du maître, attestée par ses élèves en l'absence d'une trace écrite, lui donne force et consistance. Reprenant des résultats acquis (l'associationnisme de Taine, les observations de Broca, le rôle de la mémoire développé par Ribot, les schémas de Wernicke et de Kussmaul, l'autonomie de l'idéation postulée par Broadbent), Charcot a surtout innové par une synthèse classificatoire superposant les centres corticaux, le mode de mémorisation du langage, les formes d'aphasie (une aphasie correspond à un déficit spécifique de la mémorisation) et les grands types humains (auditifs, visuels, graphiques et articulatoires). La représentation suivante en donnera le principe :

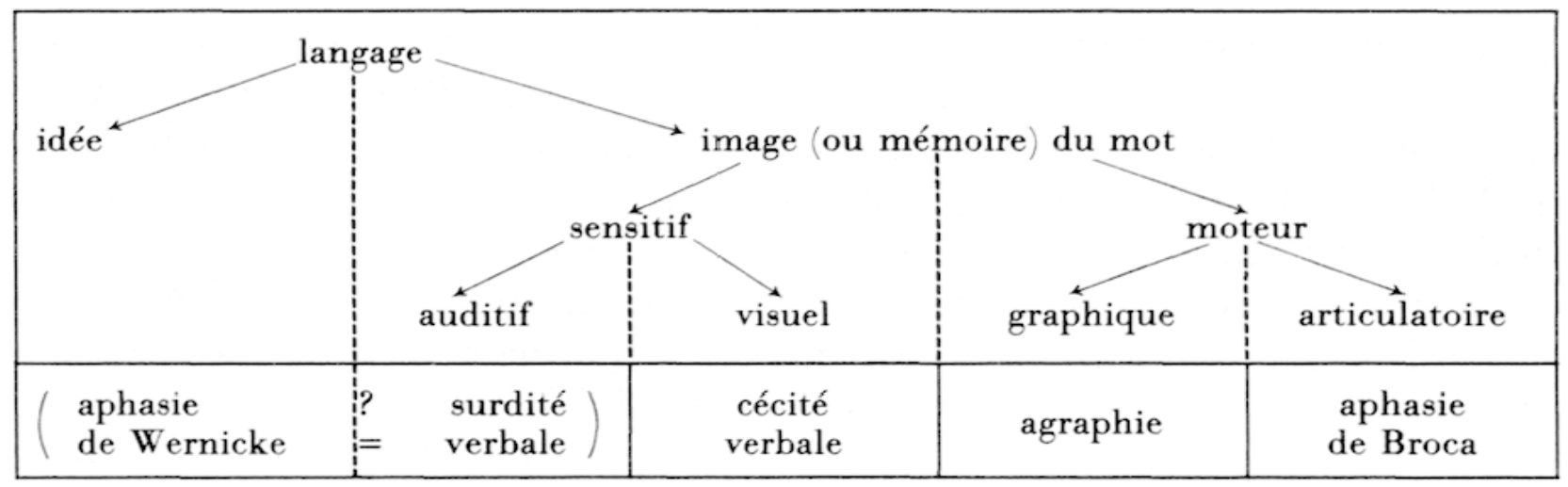

Même si certaines aphasies peuvent être associées à la perturbation de la fonction "idéatique », quatre formes en seraient assurées, provoquées par l'incapacité à articuler (aphasie de Broca); à associer les images visuelles ("cécité verbale»), auditives ("surdité verbale») ou graphiques ("agraphie») des mots. L'agraphie, au sens où elle est entendue ici, est une "découverte »de Charcot, inscrite dans l'équilibre parfait d'un schéma mais fondée, dans sa théorie, sur les plus fragiles éléments ${ }^{16}$.

Si Egger n'est jamais pris à partie explicitement, il semble qu'il ait inspiré, négativement, plus d'une fois les leçons de Charcot, obligé de se démarquer de l'anatomie et de l'expérimentation ${ }^{17}$, de poser la question de l'introspection ${ }^{18}$ et du «langage intérieur $^{19}{ }^{\text {» avant de }}$ 


\section{Gabriel Bergounioux}

récuser l'hypothèse de la "parole intérieure »- réduite à la conduite du type auditif -, au profit d'une "pensée par images » caractéristique du type visuel, celui dont Charcot se revendique et qui serait le plus répandu.

L'enseignement de Charcot, unanimement salué comme un progrès décisif, nous apparaît rétrospectivement comme une formidable régression; avec lui, le champ médical, par un retournement inattendu de ses effets, ménage, sous le nom de "centre d'idéation ", un espace spécifique qui protège le moi quand l'unité et l'autonomie de l'ego paraissaient définitivement ébranlées par la constitution de procédures de morcellement ${ }^{20}$. Paradoxe du champ scientifique : la défense du narcissisme contre les disciplines "matérialistes》 (anatomie et physiologie neurologique) ne peut plus être assurée par des doctrines philosophiques ou des humanités, dépréciées par ce qu'a produit de vérité ce qui, dans notre présent, est advenu comme "sciences humaines»; dès lors, c'était à l'adversaire de la veille, la médecine, qu'il revenait de préserver le moi, son unité (attestée par l'unité anatomique de l'individu) et l'autonomie de sa pensée (comme synthèse mémorisée des opérations sensorielles et motrices). L'émergence tardive d'une science de l'homme inversait les signes du positivisme et de l'idéalisme entre deux champs inégalement constitués : celui du savoir médical et celui du devenir de la psychologie, de la linguistique - même s'il est difficile de qualifier (ou de disqualifier) unilatéralement les théories en concurrence...

Dans le mouvement même qui préserve le moi, contre les spéculations d'Egger qui en faisait un discours historicisé et fondamentalement schizé ${ }^{21}$, Charcot doit concéder l'éclatement interne de la mémoire - présentée comme une constellation d'images - et une distribution phénoménologique en quatre "types» qui se retrouve, différemment combinée, en chaque individu; entre les deux, il peut situer le prisme irréductiblement unitaire du moi, sans contradiction avec les théories de Ribot.

\begin{tabular}{|c|c|}
\hline MÉMOIRE & TYPE \\
\hline Visuelle- & Visuel \\
\hline Auditive- & Auditif \\
\hline Graphique- & Moteur (main) \\
\hline Articulatoire & Moteur (organes vocaux) \\
\hline (Idéatique) & (Non défini) \\
\hline
\end{tabular}


Par ses subdivisions, il présente l'œuvre d'Egger comme un fragment de sa théorie, un cas parmi les cinq possibles : celui le mieux connu de l'« auditif » qui procède par l'introspection. De surcroît, il approprie à la clinique le monopole de la compétence sur la "parole», réduite à un processus sensori-moteur ${ }^{22}$.

Quant à la séduction durable que devait exercer cette théorie, il faut joindre à son formalisme rationaliste et à la netteté de la synthèse non seulement son rôle de défense du narcissisme, quand le champ scientifique dessinait les figures du sujet morcelé et du malaise dans la civilisation, mais aussi la personne de Charcot comme figure du savant et comme parole du maître :

Con questa lezione il Professore Charcot chiudeva l'anno scolastico 1883-1884 lasciando in tutti quelli che ebbero la fortuna di essere presenti, vivissimo desiderio di udire ancora la sobria et convincente parola del grande clinico della Salpêtrière ${ }^{23}$.

Dans un article publié par Binet dans la Revue des Deux-Mondes ${ }^{24}$, où sont mentionnés les ouvrages de huit auteurs, dont Egger (mais pas ceux de Charcot), nous relevons :

C'est M. Charcot qui a construit la théorie psychologique la plus complète du langage, en se servant des éléments fournis par l'aphasie $[\ldots]$ nous préférons exposer ici la théorie de $M$. Charcot, parce qu'elle embrasse tous les cas connus d'aphasie; elle est claire, complète, d'une belle ordonnance, peut-être légèrement schématique $[\ldots]$. Son principal caractère est de présenter un décalque des faits cliniques.

\section{La réputation de Charcot.}

Il n'y eut pas de véritable réponse de Victor Egger : peut-être découragé par le succès facile de Charcot (et de Ribot), peut-être déphasé par le mouvement positiviste des sciences humaines, peutêtre indifférent au devenir de la psychologie, il commet quelques articles de portée limitée sans répondre vraiment à ses détracteurs.

Quatre ans avant sa mort (1908), pourtant, et à deux reprises, il est contraint de revenir sur des hypothèses formulées un quart de siècle auparavant. D'une part, à l'occasion de la deuxième édition de $L a$ Parole intérieure, dans une préface de quelques pages, il réaffirme la constitution fondamentalement auditive de l'homme et revendique, face aux "abstractions》 de Charcot, la position expérimentale de l'observateur, inversant la caractérisation reçue des qualités du philosophe et du clinicien. 


\section{Gabriel Bergounioux}

D'autre part, répondant à un questionnaire par correspondance de Saint-Paul, un élève de Charcot, à la recherche d'une autodéfinition des «types", Egger s'emporte contre son rival, et, après avoir réaffirmé le primat de l'audition, il signe :

V. Egger, psychologue salarié par l'état, compétent en toutes ces matières ${ }^{25}$ ? ? ?

Dans le mouvement de colère qui inspire cette signature, il y a l'aveu d'un échec, l'échec d'une reconnaissance savante pour le fondateur d'une psychologie fondée sur la parole.

Pour surmonter la doctrine de Charcot, il fallut attendre la rupture philosophique du bergsonisme, commencée avec Matière et Mémoire (1896), mais surtout la critique médicale entreprise par Marie, contre les thèses de Broca et de Charcot, dans La Semaine médicale (1906). Marie rompait définitivement avec la théorie des types et l'associationnisme - c'est-à-dire avec les phases non unitaires de la psychologie du moi développée par Charcot - et, abandonnant aux philosophes et psychologues la définition du sujet, il proposait un retour à des valeurs médicales qui identifie l'aphasie à un déficit intellectuel, sans le souci des synthèses du moi.

Un élève de Marie, Moutier, pouvait annoncer deux ans plus tard :

L'aphasie fut d'emblée non seulement une maladie, mais une doctrine, et cliniciens et philosophes s'employèrent également à la disséquer et à en analyser les plus délicates nuances [...]. Ces distinctions [de Charcot], basées sur des idées théoriques touchant le mécanisme du langage intérieur, ne se vérifient jamais au lit du malade ${ }^{26}$.

Désormais, un autre partage - où la question de l'" analyse profane » n'est pas très loin - se mettait en place entre la Sorbonne et l'École de médecine, après vingt ans de domination de Charcot, sans que, du champ des sciences humaines, les hypothèses cliniques aient pu être levées, l'abandon des découvertes médicales n'étant accepté qu'après une critique radicale des médecins eux-mêmes.

\section{In fine}

Dans cette inversion des rôles entre philosophie du langage et médecine pour constituer un espace où le moi fonctionne comme unité opératoire, c'est-à-dire pour préserver le primat de la 
conscience, fút-ce au prix d'un réaménagement des instances qui le constituent (mémoire, perceptions, capacités motrices), Charcot engage intégralement les valeurs qui fondent son enseignement. Mais, pour approprier à la clinique la défense du narcissisme, il doit renoncer à l'observation et à l'expérimentation, ne soutenant son argumentation que par l'élégance réductrice des schémas, les arguments d'autorité de la citation et la mise en scène de la Salpêtrière, refusant la publication en France de propos qui ne se soutiennent pas au-delà du prestige du "pro-fesseur».

Que, parmi les sciences humaines en gestation, ce soit la philosophie - inscrite dans la tradition idéaliste française, informée des acquis de la psychologie et de la linguistique, mais s'en démarquant - qui révoque le principe du primat de la conscience indique assez quelle lacune, quel point aveugle persévérait dans le partage des sciences humaines, quand la psychologie refusait d'établir un centre de synthèse, quand la sociologie se réservait pour les faits sociaux, quand la linguistique s'interdisait la formulation d'une sémantique $^{27}$. Rejoindrons-nous Michel Foucault :

$[\ldots]$ le * je parle fonctionne comme au rebours du * je pense *. Celui-ci conduisait en effet à la certitude indubitable du Je et de son existence; celui-là au contraire recule, disperse, efface cette existence et n'en laisse apparaître que l'emplacement vide [...]. Sans doute est-ce pour cette raison que la réflexion occidentale a si longtemps hésité à penser l'être du langage : comme si elle avait pressenti le danger que ferait courir à l'évidence du « je suis »l'expérience nue du langage ${ }^{28}$ ?

Si la question du moi est posée dans le champ universitaire, c'est dans les marges non seulement des enseignements traditionnels, mais aussi des disciplines nouvelles qui prétendent parler de l'homme, de la part d'un "Victor Egger, psychologue salarié par l'état », dont la signature semble une dénégation. V. Egger est confronté à la transformation des rapports entre l'enseignement supérieur et le pouvoir républicain qui institutionnalise progressivement les sciences humaines, y trouvant une justification de son action, une sociodicée et une arme dans ses démêlés avec l'idéalisme (généralement catholique); l'idéologie officielle met au centre de ses principes la nécessité d'une morale individuelle et sociale et l'efficience de la pédagogie, deux doctrines fondées sur la « conscience " et l'apologie du moi. Une théorie comme celle d'Egger apparaissait à tout point de vue en porte à faux : idéaliste face à une philosophie officielle néo-kantienne, introspective quand le laboratoire devient le modèle de toute vali- 
dation scientifique ${ }^{29}$, antinarcissique dans une fin de siècle pédagogique. Pendant que Ribot, conforme à ces attentes, recevait une chaire au Collège de France, que la médecine s'arrogeait le privilège de la définition du sujet, l'œuvre d'Egger, en identifiant sujet et parole, ne rejoignait aucune attente mais soulevait les résistances, personnelles et collectives, qui ont effacé cette première approche de la question du sujet par le langage.

\author{
Gabriel Bercounioux \\ Université d'Orléans
}

\title{
NOTES
}

1. Pour mémoire, c'est en 1866 que Ribot fonde La Revue philosophique, qui deviendra l'organe officiel de la discipline en France même s'il lui faudra attendre 1885 pour recevoir un cours complémentaire à la Sorbonne sous l'intitulé * Psychologie expérimentale *. Pour un point de vue contemporain, on pourra se reporter à Dwelshauvers, La Psychologie française (1920).

2. Pour plus de détails, on se reportera à J. Dubois et H. Hécaen, La Naissance de la neuropsychologie du langage (1825-1865) [1969].

3. Pour illustrer ceci, que l'on songe à Cabanis au début du siècle, plus tard à Taine, et au poids démesuré que prirent, dans les revendications de préséance concernant le concept d'\& aphasie *, les arguments étymologiques, pour ne pas dire philologiques (voir ibid.).

4. C'est-à-dire l'assignation en un point donné de l'enveloppe corticale d'une * fonction * motrice ou sensorielle.

5. Sur Broca, on se reportera à Francis Schiller, Paul Broca (1979); sur les débats du XIX ${ }^{e}$ siècle concernant l'aphasie, à André Ombredane, L'Aphasie et l'Élaboration de la pensée explicite (1950).

6. Wernicke (1874), Kussmaul (1876), Lichtheim (1884), qui retrouvent à travers l'aphasie la question du moi.

7. Il prononce cette année-là une suite de conférences au Collège de France sur * Les idées latentes dans le langage > qu'il ne publiera qu'en 1897, sous une forme très remaniée, dans l'Essai de sémantique.

8. Nous avons risqué une telle interprétation dans le numéro 63 de Langue française (1984); sur la reformulation des représentations socio-politiques, on lira Claude Nicolet, L'Idée républicaine en France (1982).

9. Si Emile Egger, le père, est un des premiers à avoir su introduire la grammaire comparée en France (Grammaire comparée des langues classiques [1852]), la mère se signale aussi par sa traduction du récit de Schliemann, l'inventeur de Troie, et le frère de Victor, Max, par des traductions et une histoire de la littérature grecque.

10. Contre lequel il prend parti en 1877 dans la Revue des Deux Mondes, en réfutant sa thèse centrale : La psychologie vraiment scientifique, c'est la physiologie du cerveau [...] l'âme n'est que l'ensemble des fonctions du cerveau, et la psychologie qu'un chapitre de la physiologie. "Comme Egger le signale, * les esprits les plus éminents et les plus indépendants de l'École de médecine de Paris se sont faits les champions de cette idée ", et, s'en prenant à Luys, malgré de faibles dénégations, Egger vise ainsi, également, Vulpian et Charcot.

11. La Psychologie anglaise contemporaine de Ribot date de 1870 ; sa thèse, de 1873.

12. Ce sera Ribot, en 1889, qui recevra la première chaire de psychologie du Collège de France. 


\section{La pathologie du langage entre lettres et médecine}

13. In La Parole en public.

14. D'où son incapacité à fixer une doctrine en matière de localisations cérébrales.

15. On sait que Charcot se définissait comme un * visuel ", sa pratique de l'hypnose en dépendant pour partie, et qu'il cultivait cette réputation par sa méthode du diagnostic, ses travaux sur l'art ou sa création d'un laboratoire clinique de photographie.

16. Une partie de la démonstration (la leçon 7 dans l'édition de Rummo) manque ; ce qui reste de l'observation s'appuie sur trois cas de seconde main et sur trois cas de première main - dont deux sont déclarés non typiques, le troisième étant \& rare > selon Charcot.

17. Miliotti, p. 140 sq.

18. Ibid., p. 152.

19. Ibid., p. 167 et 177.

20. Au sens où la psychanalyse parle de corps "morcelé ".

21. Nous ne pouvons, faute de place, que renvoyer à La Parole intérieure, notamment à la deuxième partie de l'ouvrage.

22. L'œuvre d'Egger, centrée sur la parole, c'est-à-dire sur la réduction de l'histoire individuelle à la continuité intérieure de son énonciation, sera récusée par le médecin - qui ne reconnaît dans la parole qu'un ensemble fini d'opérations mentales - et restera incomprise du psychologue - dont le malentendu atteint sa limite chez Dwelshauvers (op. cit.) qui, citant Egger, parle de son ouvrage : Le Langage intérieur (sic).

23. Miliotti, op. cit., conclusion.

24. Les maladies du langage d'après les travaux récens > (1892).

25. In Le Langage intérieur et les Paraphasies (1904).

26. In L'Aphasie (1908).

27. Seule l'histoire particulière de ces sciences permettrait de comprendre la logique spécifique de ces renoncements.

28. Michel Foucault, La Pensée du dehors (1986), p. 13-14.

29. C'est Gambetta qui avait offert à Charcot ses installations de la Salpêtrière. 